\title{
INTERAÇÃO, COOPERAÇÃO E SISTEMATIZAÇÃO DA APRENDIZAGEM EM AMBIENTE VIRTUAL: UMA PROPOSTA METODOLÓGICA INOVADORA
}

Ana Paula Costa e Silva - Universidade Católica de Brasília - asilva@ucb.br Michelle Jordão Machado - Universidade Católica de Brasília - michellem@ucb.br Sandra Mara Bessa Ferreira - Universidade Católica de Brasília - sandram@ucb.br

\section{RESUMO}

Os ambientes virtuais de aprendizagem concentram diferentes recursos e ferramentas de comunicação síncrona e assíncrona, que representam um arsenal tecnológico com elevado potencial, que somente pode ser explorado em sua plenitude por meio de propostas metodológicas inovadoras. O presente trabalho descreve uma proposta de utilização de ambientes virtuais que privilegia os processos de interação, cooperação e sistematização das experiências em favor da efetividade dos processos de ensino e aprendizagem. Para comprovação de tal efetividade, são apresentados resultados de uma pesquisa realizada junto a estudantes de cursos de pós-graduação a distância, que evidenciam as contribuições que os momentos individuais e coletivos previstos na proposta metodológica aplicada podem representar para a aprendizagem dos estudantes.

Palavras-chave: ambientes virtuais de aprendizagem; interação; cooperação; sistematização.

\section{INTERACTION, COOPERATION AND LEARNING SYSTEMATIZATION IN VIRTUAL ENVIRONMENT: AN INNOVATIVE METHODOLOGY}

\begin{abstract}
The learning management systems concentrate different resources, synchronous and asynchronous communication tools, which represent a high potential technological repertoire that only can be fully explored when there are innovative methodological approaches. This work describes a form of use the learning management systems that valorize the interaction and cooperation processes, the experience systematization in favor of effectiveness of the teaching and learning processes. In order to prove this effectiveness, will be presented the results of a research with students of post-graduate distance courses. This research points the contributions that the individual and collective moments included at the applied methodological proposal can represent to the students learning.
\end{abstract}

Keywords: learning management systems; interaction; cooperation; systematization. 


\section{Contextualização}

As propostas metodológicas de cursos superiores em ambientes virtuais de aprendizagem devem levar em consideração o potencial dos processos de interação e cooperação, bem como a necessidades de momentos individuais de reflexão e sistematização das experiências, para que seja possível estabelecer um ciclo efetivo de aprendizagem.

Os ambientes virtuais de aprendizagem concentram diferentes recursos e ferramentas de comunicação síncrona e assíncrona, que representam um arsenal tecnológico com elevado potencial. No entanto, todo esse aparato tecnológico, por mais encantador que possa parecer em um primeiro momento, não garante por si só condições para os processos de interação, cooperação e aprendizagem, uma vez que depende diretamente da metodologia aplicada.

Cada vez mais, mostra-se necessário um planejamento consistente das formas de utilização de cada um dos recursos disponíveis nos ambientes virtuais, com a definição clara dos objetivos a serem alcançados. Além disso, é essencial que a utilização desses recursos seja fundamentada em uma proposta metodológica coerente com o modelo de educação e com os princípios e valores da instituição.

Ao observarmos a organização das salas de aulas virtuais, podemos identificar elementos comuns à maioria, tais como: links de acesso aos conteúdos das disciplinas, materiais complementares, bibliotecas digitais, textos de apoio etc; ferramentas de comunicação síncrona (chat e webconferência); ferramentas de comunicação assíncrona (fórum de discussão, wiki, e-mail); ferramentas de apoio (calendário, envio de atividades, notas).

O presente trabalho visa descrever uma proposta metodológica diferenciada e inovadora, baseada no pressuposto de que os processos de estudo e pesquisa individuais (imersão nos materiais didáticos), interação, aprendizagem cooperativa e sistematização das experiências são complementares e interdependentes, pois a combinação entre eles contribui para a efetividade do processo de aprendizagem dos estudantes.

\section{Explorando a recursividade nos ambientes virtuais de aprendizagem (AVA)}

A Figura 1 ilustra a interdependência positiva que se estabelece entre as diferentes etapas e momentos do processo de aprendizagem dos estudantes em ambientes virtuais de aprendizagem, com base em uma proposta metodológica que valoriza tanto os momentos individuais quanto aqueles de construção coletiva do conhecimento.

Conforme pode ser observado, o ciclo que se estabelece nessa proposta metodológica comporta a propriedade de recursividade, de forma que cada uma de suas fases/momentos pode dar início a um novo ciclo, não havendo uma linearidade obrigatória na passagem de uma fase para outra. Assim, a partir de cada fase pode surgir um ou mais ciclos, formando elos que caracterizam a recursividade.

Como exemplo, pode-se considerar uma situação em que o estudante, ao realizar pesquisas sobre determinado tema, sente necessidade de interagir com os professores e demais colegas por meio do ambiente virtual para esclarecer dúvidas ou mesmo socializar o que teve oportunidade de identificar e descobrir. Da mesma forma, ao elaborar sua Sistematização de Aprendizagem, ao final do período letivo, sentirá 
necessidade de imergir nos materiais didáticos e interagir com os professores e colegas para trocar ideias e esclarecer dúvidas que surgirem ao longo de sua elaboração.

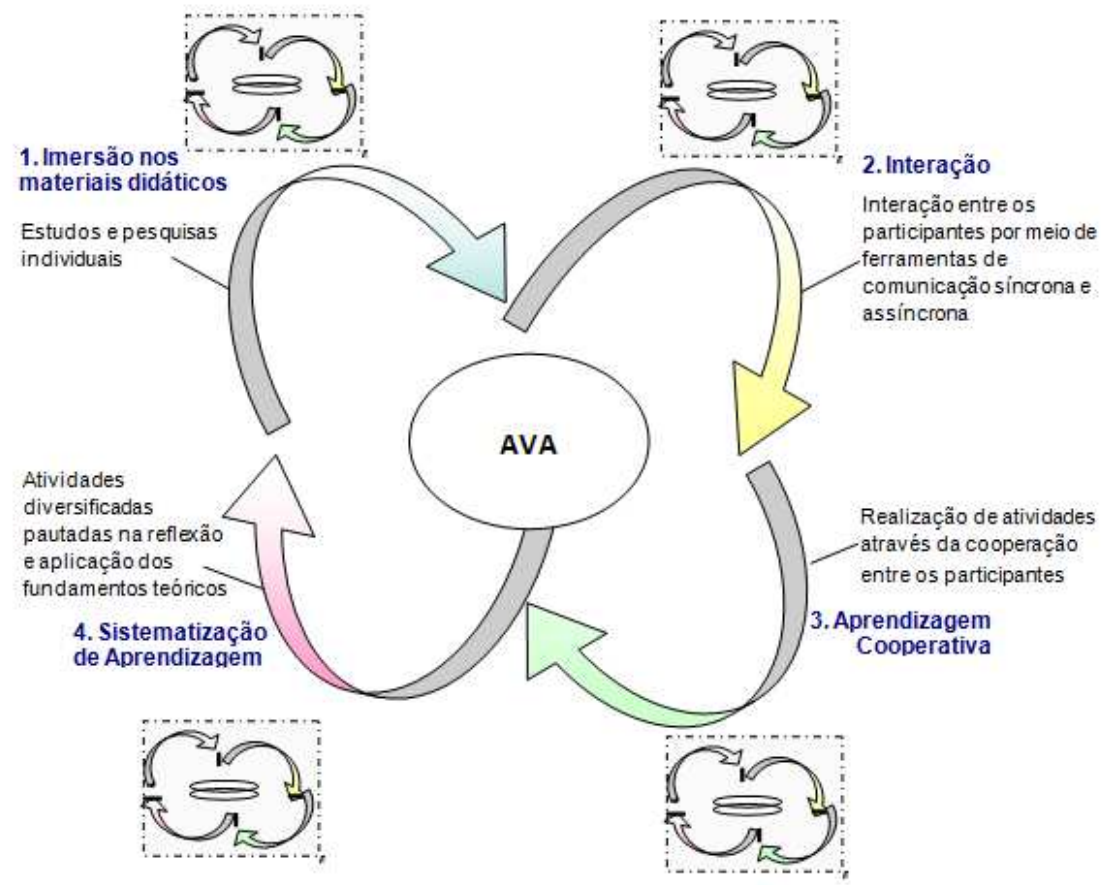

Figura 1 - Etapas da proposta metodológica em ambientes virtuais de aprendizagem

$\mathrm{Na}$ sequência serão detalhadas cada uma das fases, com suas respectivas contribuições para a aprendizagem dos estudantes.

\subsection{Imersão nos materiais didáticos: estudos e pesquisas individuais}

Tanto em cursos a distância quanto em cursos presenciais, os estudantes têm acesso aos materiais didáticos, seja na forma de livros didáticos, conteúdos hipertextuais, apostilas, ou outras formas de organização.

No caso específico de materiais hipertextuais percebe-se uma ampliação das possibilidades de desenvolvimento da autonomia do estudante na gestão de seu processo de aprendizagem. Pierre Lévy (1993) considera o hipertexto um conjunto de nós que são ligados por conexões. Tais nós podem ser palavras, expressões, imagens, vídeos, áudios, documentos complexos eu podem constituir outro hipertexto. De acordo com Lévy (1999), a hipertextualização de documentos possibilita a combinação e a indissociabilidade das funções de leitor e autor. Ele afirma que "Tudo se passa como se o autor de um hipertexto constituísse uma matriz de textos potenciais, sendo o papel dos navegadores o de realizar alguns desses textos, fazendo funcionar, cada um à sua maneira, a combinação entre os nós" (Lévy, 1999: 69). A indissociabilidade das funções de leitor e autor evidencia o potencial do hipertexto para desenvolver a autonomia do estudante, pois à medida que lê, cria possibilidades, caminhos de pesquisa, constrói sua trilha de aprendizagem.

Ao realizar leituras e se aproximar dos materiais didáticos, os estudantes avançam na construção do conhecimento, ampliam suas ideias, deparam-se com as contribuições de diferentes autores, identificam caminhos para pesquisas e V. $10 \mathrm{~N}^{\mathrm{o}}$ 1, julho, 2012 
aprofundamento dos estudos. Portanto, iniciam o processo de construção de uma base teórica e conceitual que permeará sua formação acadêmica. Os referenciais teóricos possibilitam aos estudantes elaborar intervenções substanciais, que em um segundo momento poderão impactar positivamente na efetividade da interação com os colegas e professores, que será abordada na sequência.

\subsection{Interação por meio das ferramentas síncronas e assíncronas}

A interação destaca-se como um dos eixos do processo de aprendizagem, seja na educação presencial ou a distância. Ela é propulsora da comunicação de pensamentos, problematização de ideias e apoio mútuo. As diferentes ferramentas de comunicação síncronas e assíncronas disponíveis nos ambientes virtuais de aprendizagem podem subsidiar os processos de interação entre estudantes e professores.

Ao planejar a utilização dessas ferramentas, o professor deve considerar as possibilidades e limitações de cada uma, procurando identificar aquelas mais apropriadas para alcançar os objetivos de aprendizagem em questão. De nada adianta propor a utilização de diversas ferramentas sem uma orientação clara ou um planejamento preciso, que possa servir de referência para as atividades a serem desenvolvidas no curso.

A interação entre estudantes e professores possibilita que as ações de cada sujeito modifiquem umas às outras, em um movimento constante e sistêmico, impulsionado pelo conjunto das relações entre os indivíduos.

A complexidade que caracteriza as interações em um ambiente virtual de aprendizagem requer a mediação sistemática por parte dos professores com o objetivo de potencializar as discussões e orientá-las com base na intencionalidade didática e nos objetivos de aprendizagem estabelecidos. Para que a interação se desenvolva em um grupo de forma plena é necessário que se estabeleça uma relação de diálogo e confiança.

Schutz (1989) identificou três dimensões de necessidades interpessoais que caracterizam as fases de desenvolvimento grupal: inclusão, controle e abertura (ICA), que são caracterizadas a seguir.

- Inclusão: fase inicial da interação, em que cada indivíduo sente necessidade de ser incluído no grupo, de sentir-se parte do grupo, ainda sem ter a devida clareza de seu papel e dos demais. Essa fase pode ser vivenciada de forma passiva, em que o indivíduo necessita que o incluam no grupo, ou de forma ativa, em que o indivíduo se inclui e toma iniciativa de incluir outros para que passem a pertencer ao mesmo grupo.

- Controle: fase em que os papéis são estabelecidos (líderes, liderados, brincalhões, introspectivos etc). Cada indivíduo passa a se reconhecer no grupo e a reconhecer o outro, identificando possibilidades de atuação e contribuições. Cada indivíduo procura satisfazer sua necessidade de influência e controle.

- Abertura: fase em que os indivíduos expressam sentimentos e criam vínculos afetivos. Os participantes já conseguem compreender as diferenças existentes entre seus pares e aprendem a conviver com elas e aceitá-las, o que promove a integração entre os membros.

Na última fase, o grupo já se encontra em condições de cooperar, ou seja, atuar de forma a alcançar um objetivo comum, com autonomia e responsabilidade distribuída entre os membros.

No planejamento, o professor deve levar em consideração as fases de desenvolvimento do grupo e ter habilidade para perceber e identificar o momento do 
grupo. Deve saber que leva certo tempo para que os estudantes se percebam e se reconheçam como parte de um grupo, para que sejam desenvolvidas as condições necessárias à aprendizagem cooperativa. Assim, ao elaborar as proposições de cada atividade, deve levar em consideração esse crescente do grupo e avançar gradativamente no nível de complexidade das atividades propostas.

Dentre as ferramentas de comunicação e interação mais utilizadas com a finalidade de interação e cooperação, destacam-se: fórum de discussão, wiki, chat e webconferência. Na sequência serão apresentadas algumas das possibilidades de utilização de cada uma delas.

\section{a) Fórum de discussão}

Por ser uma ferramenta de interação assíncrona, o fórum possibilita aos participantes postarem mensagens que ficam organizadas e disponíveis para acesso durante todo o período de duração da discussão. A socialização de ideias, experiências, situações-problema, indicações bibliográficas e de outros materiais cria um ambiente favorável à aprendizagem, pois viabilizar o contato dos estudantes com toda uma diversidade de contribuições que provocam reflexões sobre a sua forma de perceber e compreender os conteúdos estudados.

Para que todo o potencial do fórum para a produção coletiva de conhecimento e para a aprendizagem cooperativa seja aproveitado da melhor forma, faz-se necessária a atuação constante e sistemática do professor como moderador, instigando os estudantes a refletir e a participar. Ao professor cabe garantir o foco no tema proposto e lançar questões que possam ampliar o debate e estabelecer vínculos entre o objeto da discussão e os conteúdos estudados.

Ao elaborar a proposição para um fórum de discussão, o professor deve estar atento a questões do tipo: qual o objetivo principal do fórum? Como a proposta do fórum será comunicada aos estudantes de forma clara? Como os estudantes serão estimulados a participar? De que forma o tema proposto se articula com os objetivos de aprendizagem? Quais os resultados esperados da discussão? Quais as ações esperadas dos estudantes no fórum? Quais as ações esperadas do professor para promover a interação e a cooperação entre os participantes? Como avaliar as intervenções dos estudantes? Como elaborar feedback consistente, claro e bem fundamentado, que contribua para a aprendizagem dos estudantes?

A efetividade dos debates nos fóruns depende fortemente da atuação adequada do professor, que deve fazer intervenções de qualidade, acompanhar o debate, promover a cooperação entre os estudantes, orientá-los, mostrar-se presente e disposto a contribuir. Desde o momento da abertura do fórum deve cuidar da clareza na apresentação do enunciado. Ao longo do fórum, mostrar-se presente e fazer intervenções que contribuam para a aprendizagem dos estudantes. Ao finalizar o fórum, recomenda-se que o professor elabore uma síntese da discussão, destacando os pontos centrais que merecem maior atenção do grupo.

Cabe considerar que a efetividade dos debates depende também do engajamento dos estudantes, de suas participações frequentes com intervenções de qualidade, do caráter crítico e reflexivo de suas contribuições para o debate, de suas iniciativas de propor de novas indagações para ampliar a discussão. Portanto, a responsabilidade pela qualidade e efetividade de uma discussão no fórum é distribuída entre estudantes e professores. 


\section{b) Wiki}

Esta ferramenta de comunicação assíncrona proporciona aos estudantes uma forma simples e sistematizada de edição e registro das contribuições individuais, que podem ser visualizadas e editadas por todos os membros do grupo. A ferramenta fundamenta-se em uma dinâmica tal em que os olhares e conhecimentos de cada um se complementam, em favor de uma produção comum, compartilhada. Os membros sentem-se co-responsáveis pelos resultados a serem alcançados, uma vez que a edição é coletiva.

O grupo pode aproveitar o potencial desta ferramenta para desenvolver a produção de textos e atividades de forma cooperativa, pautada em uma lógica compartilhada pelo grupo que facilite a organização das ideias e a combinação das contribuições individuais na composição da produção coletiva.

\section{c) Chat}

Por ser uma ferramenta de comunicação síncrona, com interface simples e de fácil utilização, o chat tem se mostrado bastante útil para a comunicação informal entre estudantes e professores, com potencial para criar aproximações e fortalecer os vínculos entre os participantes na busca de objetivos comuns. Por sua dinâmica caracterizada por trocas rápidas de mensagens curtas, adéqua-se melhor a conversas livres ou discussão de assuntos mais pontuais e objetivos, como esclarecimento de dúvidas sobre atividades, organização de planos de estudo, definição de encaminhamentos e distribuição de tarefas entre os membros do grupo.

\section{d) Webconferência}

O recurso de webconferência trouxe grandes facilidades para a realização de conferências e encontros síncronos, com transmissão de áudio e vídeo, entre participantes que se encontram distribuídos geograficamente, de forma simples, através do protocolo web, bastando contar com um computador conectado à Internet, webcam, microfone e software específico. $\mathrm{O}$ que antes exigia equipamentos sofisticados e caros utilizados em videoconferências pode agora ser realizado com qualidade e reduzidos investimentos em hardware e software.

\subsection{O potencial da Aprendizagem Cooperativa}

A cooperação envolve a disposição dos estudantes para atuarem juntos na busca de objetivos comuns. Assim, a aprendizagem cooperativa somente é possível quando se tem processos de interação bem desenvolvidos e quando o grupo se encontra na fase de abertura, uma vez que cada um deverá reconhecer as possibilidades que traz para a aprendizagem do outro e também as suas limitações, que poderão ser superadas com o apoio do outro, em uma relação de interdependência positiva.

A aprendizagem cooperativa requer uma atuação constante do professor. Um requisito para o professor promover a aprendizagem cooperativa, independente das tecnologias e mídias digitais a serem utilizadas, é conhecer cada um de seus estudantes. Conhecer as possibilidades que cada um representa para o grupo e também as limitações, para que possa combinar da melhor forma os talentos, competências e habilidades individuais em favor da aprendizagem coletiva. Deve tirar proveito da 
diversidade de competências para organizar grupos heterogêneos, nos quais se amplia a perspectiva de aprendizagem com o outro, pois o grupo tende a avançar mais em seu aprendizado se é formado por pessoas com diferentes experiências culturais, em diferentes estágios de conhecimento sobre o conteúdo que será estudado e com diferentes características de comportamento.

O professor deve desenhar sua ação pedagógica com foco nos estudantes, na promoção da aprendizagem cooperativa, que é fundamental nos dias atuais, uma vez que trabalhar em equipes, de forma cooperativa, passou a ser uma exigência da contemporaneidade.

Propor que os estudantes realizem atividades de forma cooperativa é mais que uma simples estratégia para favorecer os processos de ensino e de aprendizagem. Para alcançar êxito, o educador deve considerar: o aprendiz como sujeito de sua ação; seu trabalho gerencial como mediador e facilitador da aprendizagem; as situações de aprendizagem como possibilidades para o exercício do pensamento crítico; que a comunicação e afetividade entre as pessoas envolvidas no processo pedagógico facilitam os processos de ensino e de aprendizagem.

Propor atividades desafiadoras, que possam ser realizadas em ambientes virtuais, com a utilização de diferentes tecnologias, é uma forma de sensibilizar os estudantes para a importância da cooperação na resolução de problemas complexos. Para superar a tendência ao individualismo, o professor deve criar estratégias para fazer os estudantes perceberem a importância do outro, as incompletudes de cada um, que podem ser complementadas com o que o outro traz para o grupo.

\subsection{Sistematização de Aprendizagem: um caminho para transformar realidades}

Após um processo de intensas reflexões, trocas, discussões e elaborações coletivas, é apropriado que o estudante tenha um momento reservado para sistematizar a sua aprendizagem, que representará um momento privilegiado para sua formação acadêmica.

Mas, afinal, o que é a sistematização da aprendizagem? As linhas que seguem buscam apresentar a definição, abrangência, objetivos e as possibilidades relacionadas à sistematização de aprendizagem.

A sistematização da aprendizagem tem relação com a experiência de aprendizagem vivenciada ao longo de cada disciplina, bem com a incorporação dessas aprendizagens às práticas profissionais e formação acadêmica. Nesse contexto, a sistematização concentra um elevado potencial para a prática transformadora da realidade em que o sujeito (estudante) está inserido, pois ele desenvolve a atividade na perspectiva de articular o presente, utilizando-se de toda a fundamentação teórica, das reflexões críticas, debates e conhecimentos construídos de forma cooperativa; com o vir a ser, com possibilidades futuras e potencialidades.

$\mathrm{O}$ momento individual reservado à sistematização de aprendizagem do estudante cria condições para que ele organize o seu pensamento, ordene os conhecimentos até então construídos, identifique suas inquietações, avanços, percepções, interpretações, intenções, bem como os pontos de convergência e divergência com os conteúdos estudados. Com isso, tem condições de reconstruir o processo vivenciado na experiência e avançar em direção à elaboração de proposições.

As múltiplas possibilidades da sistematização de aprendizagem podem ser sintetizadas em: ter uma compreensão mais profunda das experiências realizadas; 
estabelecer reflexões teóricas com vistas a articular as teorias estudadas com os conhecimentos construídos e sua aplicação em contextos práticos; registrar, por meio de atividades diversificadas, suas interpretações, percepções, ideias e construções de forma organizada e sistematizada, com vistas à socialização da experiência e consultas futuras ao longo da formação acadêmica.

$\mathrm{Na}$ dinâmica do ciclo de aprendizagem em ambiente virtual, a sistematização de aprendizagem impulsionará pesquisas, estudos e ampliação das abordagens teóricas, criando condições para a construção de conhecimentos nas demais fases do ciclo.

\section{O que revela a pesquisa de autoavaliação dos estudantes}

Com o intuito de comprovar o potencial da presente proposta metodológica em ambiente virtual para a aprendizagem do estudante, são apresentados resultados da pesquisa de satisfação dos estudantes com as unidades de estudo. Tal pesquisa foi realizada em dezembro de 2011, com um total de 85 estudantes de 15 cursos de pósgraduação a distância, por meio de questionário eletrônico, que concentra as seguintes avaliações: Avaliação do Conteúdo da Unidade de Estudo; Avaliação do Desempenho do Professor; e Autoavaliação do Estudante. Para responder às avaliações, os estudantes utilizaram a seguinte escala de concordância: 1. Discordo Totalmente; 2. Discordo Parcialmente; 3. Nem discordo e nem Concordo; 4. Concordo Parcialmente; 5. Concordo Totalmente.

Em função do objetivo do trabalho, são apresentados somente os dados referentes à dimensão "Autoavaliação do Estudante", que concentra os 11 itens listados na figura a seguir, com as respectivas médias obtidas.

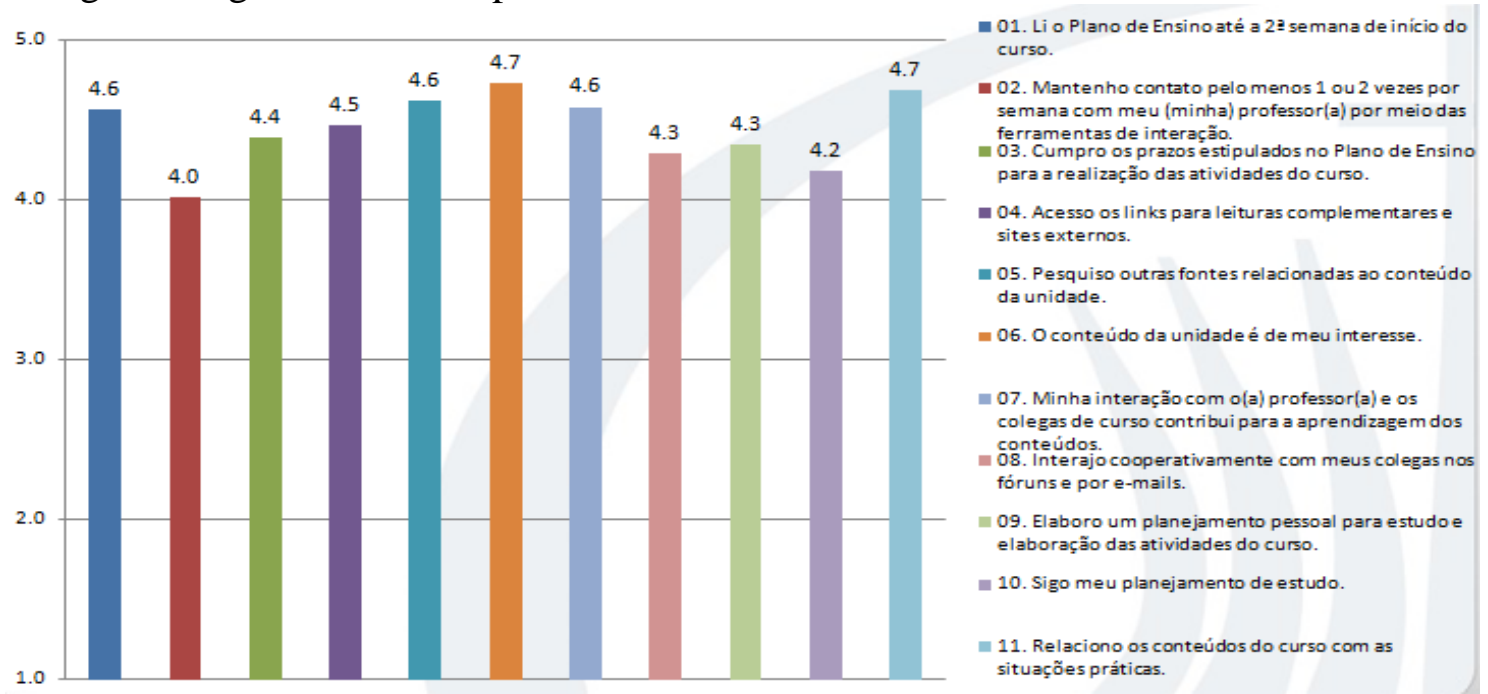

Tabela 1 - Valor médio conferido pelos estudantes no processo de Auto-avaliação

$\mathrm{Na}$ análise, observou-se que não há uma diferença significativa entre as médias obtidas nos itens avaliados. O resultado conduz a uma avaliação positiva da atuação do estudante nos cursos de graduação no ambiente virtual. É possível identificar uma relação direta de vários itens com as fases propostas no ciclo de aprendizagem em ambiente virtual. Os itens 4, 5 e 6 relacionam-se diretamente com o momento individual, de estudo, pesquisa e imersão nos materiais didáticos hipertextuais. A coresponsabilidade e cooperação passam a ser atitudes básicas dos estudantes que necessitam ser cultivadas nos ambientes educacionais, em todos os níveis e etapas V. $10 \mathrm{~N}^{\mathrm{o}} 1$, julho, 2012 
processuais. Daí a necessidade de maior ênfase nas estratégias cooperativas de aprendizagem, nas colaborações mútuas e na valorização de propostas coletivas e um maior cuidado com as questões éticas.

Dessa forma, podemos inferir que os AVAs permitem uma interatividade tendo a intenção de se construir uma aprendizagem colaborativa. Os hipertextos ganham importância nesse contexto de rede, pois potencializam ligações espaciais e temporais diferenciadas da linearidade na medida em que se conectam textos à outros textos, gerando polifonia de sentidos.

Cabe ressaltar ainda que a presença, o planejamento individual e a disciplina do estudante no ambiente virtual são requisitos necessários para que essa aprendizagem colaborativa aconteça, conforme indicam os itens 1, 2, 3, 8 e 9. O estudante, nessa perspectiva, é visto como aquele que conhece, que aprende, que identifica, que planeja sua aprendizagem e a anuncia aos demais.

$\mathrm{O}$ item 7, em que se tem uma das médias mais altas, relaciona-se com a fase de interação dos estudantes com seus colegas e professor, em que os estudantes reconhecem uma contribuição direta para a aprendizagem dos conteúdos. O item 8 tem relação com a aprendizagem cooperativa que se estabelece entre os estudantes por meio das ferramentas de comunicação. Assim, percebe-se que o estudante é dimensionado como o responsável por sua aprendizagem, construtor do conhecimento, provedor de sentido.

Por fim, o item 11, com a média mais alta, evidencia a contribuição do momento individual de Sistematização da Aprendizagem, para que o estudante possa relacionar os conteúdos estudados com situações práticas vivenciadas. A sistematização incentiva um constante diálogo entre saberes: uma articulação criadora entre o saber cotidiano e os conhecimentos teóricos, que se relacionam e se alimentam mutuamente. Permite ao estudante perceber que as teorias não são absolutas ou definitivas, pois estão em constante reconstrução e recriação criativa por meio das intervenções dos vários sujeitos, uma vez que se colocam a serviço da prática transformadora e de seus inéditos desafios (Holliday, 2006).

\section{Conclusão}

A educação deve se constituir como um processo tipicamente interativo, cooperativo e dialógico. Para que isso ocorra em sua essência, deve existir uma relação positiva entre os membros do grupo, o educando deve se dar conta que seu saber é importante para o conjunto de saberes do restante do grupo. Aprende-se com o outro numa posição de diálogo, que pressupõe saber escutar, elaborar perguntas e estar aberto para o novo e para o diferente que o outro carrega consigo, e que pode desestabilizar o que já tem consolidado em seu saber. Nesta perspectiva, o outro passa de um aparente limite ou dificuldade à possibilidade de crescimento e aprendizagem.

Para Mailhiot (1998), a construção de um ambiente de aprendizagem propício à cooperação só é possível a partir de relações interpessoais baseadas na aceitação, na interdependência e na complementariedade. É importante que haja preocupação e ação no sentido de tornar os ambientes de aprendizagem cooperativa locais de construção de conhecimentos e de trocas afetivas e efetivas. Neste sentido, os ambientes virtuais de aprendizagem, as ferramentas da web 2.0, as redes sociais e outros recursos tecnológicos podem se tornar poderosos aliados dos professores. 


\section{Referências Bibliográficas}

HOLliDAY, O. J. Para sistematizar experiências. Tradução de Maria Viviana V. Resende. $2^{\text {a }}$ edição revista. Brasília: MMM, 2006.

LÉVY, P. As Tecnologias da Inteligência. Rio de Janeiro: Ed. 34, 1993.

LÉVY, P. Cibercultura. São Paulo: Editora 34, 1999.

MAILHIOT, G. B. Dinâmica e Gênese dos Grupos. São Paulo: Duas Cidades, $8^{a}$ Edição, 1998.

OLIVEIRA, M. N. Lewin, Bion e Schutz: um olhar a respeito de fases. Disponível em: http://www.processosgrupais.com.br/fases_txt.asp. Acesso em 10 de abril de 2012.

SCHLEMMER, E. Inovações? Tecnológicas? na Educação. In: MILL, Daniel Ribeiro Silva; PIMENTEL, Nara Maria (Org.). Educação a distância: desafios contemporâneos. São Paulo: UdUFSCar, 2010.

SCHUTZ, Will. Profunda simplicidade. São Paulo: Agora. 1989. 